Estela M. L. Aquino 1

\title{
Adolescência e reprodução no Brasil: a heterogeneidade dos perfis sociais
}

Maria Luiza Heilborn 2

Daniela Knauth 3

Michel Bozon 4

Maria da Conceição Almeida 1

Jenny Araújo 1

Greice Menezes 1

\author{
Adolescence and reproduction in Brazil: \\ the heterogeneity of social profiles
}

${ }^{1}$ MUSA - Programa de Estudos em Gênero e Saúde, Instituto de Saúde Coletiva, Universidade Federal da Bahia. Rua Padre Feijó 29, 4 a andar, Salvador, $B A$ 40110-170, Brasil. musa@ufba.br

2 Programa em Gênero, Sexualidade e Saúde, Instituto de Medicina Social, Universidade do Estado do Rio de Janeiro.

Rua São Francisco Xavier 524 Bloco E, Rio de Janeiro, $R J$ 20550-013, Brasil.

sexgen@uerj.b

3 Núcleo de Pesquisa em Antropologia do Corpo e da Saúde, Universidade Federal do Rio Grande do Sul. Av. Bento Gonçalves 9500, Prédio 43.311, Bloco A2, Porto Alegre, RS 91509-900, Brasil. nupacs@ifch.ufrgs.br 4 Institut National d'Études Démographiques. 133 Boulevard Davout, 75020 Paris, France. ined@ined.fr
Abstract This study aims to estimate the prevalence of adolescent pregnancy (AP) in three Brazilian cities - Salvador, Rio de Janeiro, and Porto Alegre-and to examine the profiles of pregnant girls and their partners in relation to pregnancy outcomes. Data for a retrospective assessment of AP were collected using an interview-based household survey applied to a stratified sample of males and females aged 18-24. A total of 4,634 individuals were interviewed (85.2\% of the eligible individuals). 21.4\% of males and 29.5\% of females aged 20-24 reported AP, but few such pregnancies had taken place before age 15 (0.6\% and 1.6\%). Pregnancy between adolescent partners was reported by $55.1 \%$ of males and $27.9 \%$ of females. $79.8 \%$ of the latter became pregnant while involved in a stable relationship with an older partner. AP levels varied inversely to schooling and income levels. $72.2 \%$ of females and $34.5 \%$ of males carried their first AP to completion, and more cases of induced abortion were reported by male partners in pregnancies (41.3\%) than by females (15.3\%). With the birth of the first child, 25.0\% of women interrupted their studies temporarily and $17.3 \%$ definitively. However, $42.1 \%$ of primiparous mothers were already outside school before pregnancy.

Key words Gender; Pregnancy in Adolescence; Reproductive Health; Sexuality

Resumo Neste estudo, estimou-se a prevalência de gravidez na adolescência (GA), em Salvador, Rio de Janeiro e Porto Alegre, analisando-se o perfil de quem engravida e seus parceiros e os resultados da gestação. Trata-se de inquérito domiciliar, com entrevistas de uma amostra estratificada de homens e mulheres entre 18 e 24 anos, para a avaliação retrospectiva da GA. Foram entrevistados 4.634 jovens (85,2\% dos elegíveis); 21,4\% dos homens e 29,5\% das mulheres com 20 anos e mais referiram GA, mas poucas se deram antes dos 15 anos (0,6\% e 1,6\%). A gravidez entre adolescentes foi relatada por 55,1\% dos homens e 27,9\% das mulheres; a maioria dessas teve a GA em relacionamento estável com parceiro mais velho (79,8\%). A ocorrência de GA variou inversamente com a escolaridade e a renda. A primeira GA foi levada a termo por $72,2 \%$ das mulheres e 34,5\% dos homens, estes com maior percentual de relato de aborto provocado (41,3\% contra 15,3\% das moças). Com o nascimento de um filho antes dos 20 anos, parte das moças parou os estudos temporária (25,0\%) ou definitivamente (17,3\%), mas 42,1\% já se encontravam fora da escola.

Palavras-chave Gênero; Gravidez na Adolescência; Saúde Reprodutiva; Sexualidade 


\section{Introdução}

A adolescência tem despertado grande interesse, tanto na mídia, quanto no âmbito das políticas públicas. Especialmente a partir de 1985, definido pela Organização das Nações Unidas (ONU) como Ano Internacional da Juventude, inúmeras iniciativas foram desencadeadas em todo o mundo, visando o levantamento das necessidades sociais dos jovens que viriam a constituir as futuras gerações de adultos no terceiro milênio. Esse processo de institucionalização refletiu mudanças que vinham ocorrendo quanto às expectativas sociais diante dessa etapa da vida, no sentido de reservá-la prioritariamente aos estudos, com vistas a capacitar os jovens sujeitos para o ingresso em melhores condições no mercado de trabalho (Sabóia, 1998).

$\mathrm{Na}$ área de saúde, a delimitação de necessidades dos jovens tem se apoiado em uma definição de adolescência de base etária (10 aos 19 anos), período que se considera como caracterizado por grandes transformações físicas, psicológicas e sociais (WHO, 1986). Freqüentemente, incorre-se em uma naturalização do processo de transição da infância à vida adulta e, ao mesmo tempo, reitera-se o caráter "imaturo" e "irresponsável” dos jovens (Stern \& Garcia, 1999), elegendo-se como temas prioritários o uso abusivo de drogas, os acidentes de trânsito e as violências, as doenças sexualmente transmissíveis e as gestações não planejadas (MS, 1999).

Contudo, a gravidez na adolescência não é um fenômeno recente. Historicamente, as mulheres vêm tendo filhos nessa etapa e, mesmo em um contexto de intensa redução da fecundidade, não se constatou no Brasil um deslocamento correspondente da reprodução para faixas etárias mais velhas, tal como ocorreu em países industrializados centrais. A maioria das mulheres brasileiras vem tendo, em média, dois filhos e parte significativa delas tem encerrado precocemente suas carreiras reprodutivas por meio de uma laqueadura tubária (BEMFAM/ DHS, 1997). Nesse contexto demográfico, a gravidez na adolescência passa a ter grande visibilidade social, principalmente ao se exibirem os dados do Sistema Nacional de Nascidos Vivos (SINASC), onde se observa um aumento relativo dos nascimentos de mães com menos de vinte anos, já que por meio dessa base não se produzem imediatamente taxas populacionais.

A abordagem do tema da gravidez na adolescência tem enfatizado o caráter de problema social do fenômeno, partindo do pressuposto de que nas adolescentes existiria "incapacidade fisiológica para gestar e incapacidade psicológica para criar" (Camarano, 1998:44). A gestação é encarada necessariamente como indesejável, com conseqüências biológicas, psicológicas e sociais negativas (Brandão, 2003).

Os estudos demográficos, psicossociais e epidemiológicos são freqüentemente restritos às mulheres, reforçando a invisibilidade social do parceiro masculino (Lyra, 1998), seja ele adolescente ou não. A homogeneização da "adolescente grávida”, em um país de grande heterogeneidade social e regional, traduz-se em um discurso moral e regulador que coloca as jovens mulheres como vítimas da própria ignorância ou inconseqüência, resultando em políticas voltadas ao controle da "gravidez precoce" (MS, 1999).

Estudos recentes têm criticado o enfoque de risco que permeia a literatura sobre sexualidade e reprodução na adolescência, procurando evidenciar a complexidade do fenômeno e os desafios colocados para sua adequada investigação (Brandão, 2003; Le Van, 1998; Stern \& Medina, 2000). Quanto às implicações sobre as trajetórias escolares e profissionais das jovens mulheres, os autores argumentam que grande proporção de gestações na adolescência acontece depois que as jovens já deixaram a escola, não podendo, portanto, ser consideradas como determinantes da pobreza, mas possivelmente por ela condicionadas (Hoffmann, 1998; Stern \& Medina, 2000).

No presente estudo, a juventude é compreendida como processo de transição da infância à vida adulta, com progressiva emancipação da família de origem e da escola (Heilborn et al., 2002). A aquisição de autonomia material e residencial ocorre de maneira mais tardia, atualmente, por meio da obtenção de um emprego e da constituição de uma unidade conjugal e/ou familiar própria (Galland, 1995). A experiência de uma gravidez inscreve-se em uma etapa de aprendizado da sexualidade, que assume contornos singulares no contexto da cultura sexual brasileira, envolvendo complexas interações entre homens e mulheres, o que torna necessário situá-la no quadro das relações e papéis de gênero (Heilborn et al., 2002). Temse como pressuposto que a gravidez na adolescência é vivida de múltiplas formas e que os contextos sociais definem universos de possibilidades e de significações diferentes entre os jovens de distintas classes sociais.

Trata-se de um estudo multicêntrico, envolvendo estratégias metodológicas qualitativas e quantitativas, realizado em três capitais brasileiras - Salvador, Rio de Janeiro e Porto Alegre - que apresentam contextos socioculturais distintos. Na primeira etapa, foram realizadas entrevistas semi-estruturadas com jovens das três cidades (Brandão et al., 2001; Heilborn 
et al., 2002), cujos resultados embasaram a formulação de hipóteses e a construção de um questionário aplicado na etapa subseqüente, por intermédio de um inquérito domiciliar de amostras representativas das mesmas cidades. Neste artigo são apresentados os primeiros resultados da etapa quantitativa, traçando um panorama da ocorrência do fenômeno da gravidez na adolescência e de seus resultados. Pretendeu-se, portanto, estimar sua magnitude, analisar as características de quem engravida e de seus parceiros, explorar determinantes sociais do fenômeno e conseqüências do nascimento de um filho nessa etapa da vida.

\section{Material e métodos}

A etapa quantitativa do estudo, descrita aqui, envolveu um inquérito domiciliar, com amostra probabilística estratificada em três estágios, de homens e mulheres entre 18 e 24 anos, moradores em Salvador, Rio de Janeiro e Porto Alegre. A decisão de não focalizar diretamente a faixa etária de 10 a 19 anos sustenta-se no objetivo de inquirir sobre os desdobramentos de uma gravidez na adolescência em momento posterior, mas suficientemente próximo ao evento para assegurar uma boa rememoração. Com base na estimativa de que, em 1996, aos 18 anos de idade, $25,3 \%$ das mulheres brasileiras já tinham experimentado pelo menos uma gravidez (BEMFAM/DHS, 1997), o tamanho da amostra foi definido em 1.500 entrevistas para cada um dos municípios estudados, num total de 4.500. Inicialmente, foi procedida a estratificação de setores censitários (SC) de cada município, agrupando-os em cinco estratos, segundo indicadores da situação sócio-econômica dos seus habitantes (renda média do chefe do domicílio e proporção de chefes com doze ou mais anos de escolaridade). A constituição da amostra de setores foi feita independentemente em cada estrato, por meio de uma seleção aleatória proporcional ao número de jovens com idade entre 18 e 24 anos em cada setor. A seguir, em cada SC selecionado, foi produzida uma listagem de todos os domicílios particulares permanentes nos quais existiam moradores com idade de 18 a 24 anos completos na data de referência da pesquisa, definida como 31 de julho de 2001. Com base nessa listagem, em cada setor foram sorteados 33 domicílios, com eqüiprobabilidade e sem reposição, em cada um dos quais selecionou-se aleatoriamente um jovem para ser entrevistado.

Os dados foram produzidos mediante a aplicação de questionário padronizado em entre- vistas face a face, realizadas até a quarta visita por entrevistadores de curso superior em Ciências Humanas, com idade até trinta anos, treinados para este fim. O questionário foi construído baseando-se em análise dos resultados da etapa qualitativa, reconstruindo as trajetórias escolares, profissionais e afetivo-conjugais dos jovens. Em razão da complexidade e do caráter pouco linear das duas primeiras, a estruturação do instrumento baseou-se na trajetória afetivo-conjugal (incluindo a sexualidade e a reprodução), relacionando os principais eventos com as situações escolares, profissionais e residenciais. Foram privilegiados os primeiros eventos sexuais e reprodutivos e aqueles mais recentes, sempre considerados por referência a relacionamentos para os quais foram descritos o contexto e os protagonistas.

O controle de qualidade envolveu procedimentos de supervisão do trabalho de campo, a revisão da totalidade dos questionários e o reteste de uma subamostra de $20 \%$ destes, efetuada de forma independente por outra pessoa.

$\mathrm{Na}$ análise de dados, a amostra foi ponderada com base nas probabilidades com que foram selecionadas as unidades de cada estágio correspondente. A medida de ocorrência de gravidez foi a prevalência, para cujo coeficiente estimou-se intervalo de confiança a $95 \%$. A prevalência de gravidez na adolescência por local de residência é apresentada segundo três pontos de corte etário: antes dos 20, dos 18 e dos 15 anos. Para o cálculo da prevalência bruta de gravidez na adolescência antes dos vinte anos, foram excluídos os jovens de 18 e 19 anos, que em razão da idade poderiam ainda vir a experimentar um episódio reprodutivo antes dos vinte anos. Para fins de comparação, nas três faixas são apresentadas as medidas de prevalência sobre a população total (inclusive virgens) e entre os que já tinham iniciado sua vida sexual. As diferenças da ocorrência entre estratos específicos foram testadas pelo $\chi^{2} \mathrm{de}$ Pearson.

A pesquisa atendeu a todos os princípios éticos requeridos, tendo sido aprovada pelos Comitês de Ética das três universidades brasileiras envolvidas no projeto.

\section{Resultados}

\section{População do estudo}

Nas três cidades, foram listadas 50.052 unidades, em 171 setores censitários, das quais $94,5 \%$ eram domicílios particulares permanentes. Exceto $2 \%$ desses, sobre os quais não se obteve in- 
formações, 25,4\% dos demais possuíam jovens na faixa etária de interesse, constituindo uma base de 5.441 domicílios elegíveis, sobre a qual se alcançou uma taxa de resposta de $85,2 \%$. O reteste de $20 \%$ das entrevistas resultou em concordância acima de $96 \%$ das respostas quanto aos eventos selecionados para o controle de qualidade, entre os quais incluía-se a experiência prévia de relação sexual, gravidez e filhos.

Foram entrevistados 4.634 jovens $(47,2 \%$ homens e $52,8 \%$ mulheres) dentre os quais, respectivamente, $93,0 \%$ e $81,6 \%$ já tinham se iniciado sexualmente, sendo a idade mediana da iniciação sexual de 16,2 anos para os rapazes e 17,9 anos para as moças. A distribuição de renda revelou-se bastante desigual nas três cidades e $36,2 \%$ dos jovens declararam uma renda familiar per capita menor ou igual a $\mathrm{R} \$ 180,00$, que correspondia a um salário mínimo nacional à época da pesquisa. Entretanto, essa proporção foi de 25,5\% em Porto Alegre, 27,9\% no Rio de Janeiro e 56,3\% em Salvador ( $p=0,0000$ ). A escolaridade dos jovens também variou intensamente, confirmando marcadas desigualdades regionais; assim, tomando-se os indivíduos de 20 a 24 anos que já tinham alcançado nível superior, mesmo incompleto, os percen- tuais foram de 34,8\% em Porto Alegre, 26,4\% no Rio de Janeiro e 14,8\% em Salvador. As trajetórias escolares das mulheres mostraram-se um pouco mais longas que as dos homens, com as primeiras completando mais freqüentemente pelo menos o segundo grau - respectivamente, $61,1 \%$ e $50,3 \%(p=0,0014)$.

Gravidez na adolescência:

a magnitude do fenômeno

A experiência de gravidez antes dos vinte anos foi relatada por $21,4 \%$ dos homens e $29,5 \%$ das mulheres que já haviam ultrapassado essa idade, percentuais que aumentam um pouco quando se considera no denominador apenas os que já haviam tido iniciação sexual (Tabela 1). Entretanto, adotando-se como ponto de corte os 18 anos - que constituem o marco da maioridade civil brasileira - a prevalência observada é bem mais baixa, sendo relatada por $8,9 \%$ dos homens e $16,6 \%$ das mulheres. Antes dos 15 anos, os valores são pequenos, situando-se em $0,6 \%$ dos rapazes e $1,6 \%$ das moças. A gravidez entre adolescentes - que merece ser distinguida das demais ocorrências reprodutivas foi relatada por $55,1 \%$ dos homens e $27,9 \%$ das

Tabela 1

Prevalência bruta de gravidez antes dos 20, 18 e 15 anos, segundo cidade e sexo.

Porto Alegre, Rio de J aneiro e Salvador, Brasil, 2000

\begin{tabular}{|c|c|c|c|c|c|c|c|c|c|c|c|c|}
\hline \multirow{3}{*}{$\begin{array}{l}\text { Sexo/Faixa etária } \\
\text { (anos) }\end{array}$} & \multicolumn{12}{|c|}{ Prevalência de gravidez* } \\
\hline & \multicolumn{3}{|c|}{ Porto Alegre } & \multicolumn{3}{|c|}{ Rio de J aneiro } & \multicolumn{3}{|c|}{ Salvador } & \multicolumn{3}{|c|}{ Total } \\
\hline & $\mathrm{n}$ & $\%$ & IC $95 \%$ & $\mathrm{n}$ & $\%$ & IC $95 \%$ & $\mathrm{n}$ & $\%$ & IC $95 \%$ & $\mathrm{n}$ & $\%$ & IC $95 \%$ \\
\hline \multicolumn{13}{|l|}{ Feminino } \\
\hline \multirow[t]{2}{*}{ Antes de $20^{* *}$} & 475 & 27,7 & $20,5-36,2$ & 514 & 29,0 & $23,8-34,8$ & 623 & 31,3 & $26,1-37,1$ & 1612 & 29,5 & $26,3-33,0$ \\
\hline & 452 & 29,1 & $21,8-37,7$ & 453 & 32,2 & $26,4-38,5$ & 525 & 37,8 & $31,7-44,4$ & 1430 & 33,4 & $29,8-37,3$ \\
\hline \multirow[t]{2}{*}{ Antes de 18} & 700 & 13,5 & $9,8-18,3$ & 803 & 16,0 & $12,8-19,8$ & 932 & 19,0 & $15,3-23,3$ & 2435 & 16,6 & $14,5-18,9$ \\
\hline & 631 & 14,8 & $10,8-19,9$ & 646 & 19,6 & $15,8-24,0$ & 714 & 24,8 & $20,2-30,1$ & 1991 & 20,4 & $17,9-23,1$ \\
\hline \multirow[t]{2}{*}{ Antes de 15} & 700 & 2,2 & $1,2-3,8$ & 803 & 1,2 & $0,6-2,4$ & 932 & 3,5 & $2,2-5,5$ & 2435 & 1,6 & $1,4-2,9$ \\
\hline & 631 & 2,4 & $1,3-4,2$ & 646 & 1,5 & $0,8-2,9$ & 714 & 4,5 & $2,8-7,3$ & 1991 & 2,5 & $1,8-3,6$ \\
\hline \multicolumn{13}{|l|}{ Masculino } \\
\hline \multirow[t]{2}{*}{ Antes de $20^{* *}$} & 473 & 18,4 & $12,2-26,9$ & 476 & 20,6 & $16,2-25,9$ & 465 & 24,4 & $19,4-30,3$ & 1414 & 21,4 & $18,3-24,9$ \\
\hline & 455 & 19,4 & $12,9-28,0$ & 461 & 21,5 & $16,7-27,1$ & 444 & 26,0 & $20,7-32,0$ & 1360 & 22,4 & $19,1-26,2$ \\
\hline \multirow[t]{2}{*}{ Antes de 18} & 735 & 8,4 & $6,0-11,5$ & 714 & 7,5 & $5,1-10,8$ & 726 & 12,1 & $9,4-15,5$ & 2175 & 8,9 & $7,2-11,0$ \\
\hline & 694 & 8,9 & $6,4-12,3$ & 672 & 7,9 & $5,4-11,6$ & 667 & 13,4 & $10,2-17,3$ & 2033 & 9,6 & $7,7-12,0$ \\
\hline \multirow[t]{2}{*}{ Antes de 15} & 735 & 0,6 & $0,2-1,4$ & 714 & 0,5 & $0,2-1,4$ & 726 & 0,9 & $0,2-4,0$ & 2175 & 0,6 & $0,3-1,4$ \\
\hline & 694 & 0,6 & $0,2-1,5$ & 672 & 0,6 & $0,2-1,5$ & 667 & 1,0 & $0,2-4,5$ & 2033 & 0,7 & $0,3-1,6$ \\
\hline
\end{tabular}

* Na linha superior de cada célula, toda a população na faixa etária, cidade e sexo correspondente.

$\mathrm{Na}$ linha inferior, em itálico, excluídos os virgens.

** Excluídos aqueles com idade inferior a 20 anos. 
mulheres (dados não apresentados). Estas, em sua maioria, experimentaram a primeira gravidez com um parceiro mais velho $(37,6 \%)$ ou muito mais velho $(42,2 \%)$; mais freqüentemente, na ocasião da primeira gravidez, elas encontravam-se morando com o parceiro, com ou sem familiares $(21,9 \%$ em contraste com $7,8 \%$ dos homens entrevistados) (Tabela 2). Todavia, em ambos os grupos, a maior parte das gestações, em sua primeira vez, se deu fora de uma união conjugal: $86,6 \%$ dos homens e $74,2 \%$ das mulheres, ao engravidarem antes dos vinte anos, moravam ainda com suas famílias de origem.

É preciso destacar que as gestações ocorreram no contexto de relacionamentos afetivos estabelecidos, sendo pequeno o percentual de homens $(14,2 \%)$ e quase desprezível o de mulheres $(2,5 \%)$ cujo primeiro episódio aconteceu em uma parceria eventual (Tabela 2). A maioria das moças $(56,2 \%)$ tinha como parceiro da primeira gestação antes dos vinte anos aquele com quem se iniciaram sexualmente, em contraste com $21,0 \%$ dos rapazes na mesma condição.

$\mathrm{Na}$ primeira gravidez adolescente, a ampla maioria dos jovens entrevistados $(85,6 \%$ dos homens e $70,3 \%$ das mulheres) não estava pretendendo engravidar, ou sequer pensava no assunto; porém, somente $36,3 \%$ dos rapazes e $31,4 \%$ das moças declararam estar em uso de contracepção por essa ocasião (Tabela 2). Esses percentuais foram mais baixos do que aqueles declarados quanto ao uso de contracepção na primeira relação sexual (respectivamente, $54,5 \%$ e $52,5 \%$ ), ressaltando igualmente a mudança no perfil contraceptivo: na iniciação sexual, $81,6 \%$ dos jovens que referiram contracepção fizeram uso de preservativo masculino, o qual se reduz para $42,2 \%$ por época da primeira gravidez antes dos vinte anos, quando o método mais citado foi a pílula $(43,4 \%$ ) (dados não apresentados). Contudo, 15,6\% das mulheres declararam que, nessa época, estavam tentando engravidar (Tabela 2). Mais ainda, 72,2\% de todas as que engravidaram antes dos vinte anos levaram a primeira gestação a termo, mesmo que a decisão tenha envolvido conflitos e negociações com seus parceiros e familiares. Ao contrário, os homens, majoritariamente, relataram que, antes dos vinte anos, na primeira vez que engravidaram uma parceira, a gestação foi interrompida por meio de um aborto provocado $(41,4 \%)$ ou espontâneo $(18,4 \%)$ (Tabela 2$)$. Como resultado de experiências tão distintas, entre aqueles que tinham experimentado pelo menos uma gravidez na adolescência, 50,9\% dos homens e apenas $16,5 \%$ das mulheres mantinham-se sem filhos por ocasião da entrevista (dados não apresentados).
Tabela 2

Distribuição de homens e mulheres com gravidez antes dos 20 anos, segundo situação no primeiro episódio. Porto Alegre, Rio de J aneiro e Salvador, Brasil, 2000.

\begin{tabular}{|c|c|c|c|}
\hline Características & $\begin{array}{c}\text { Mulheres } \\
\%\end{array}$ & $\begin{array}{c}\text { Homens } \\
\%\end{array}$ & p-valor \\
\hline Tipo de parceria & $n=682$ & $\mathrm{n}=377$ & \\
\hline Parceiro/a da iniciação sexual & 56,3 & 21,0 & 0,0000 \\
\hline O utro/a parceiro/a fixo/a* & 41,1 & 64,8 & \\
\hline Parceiro/a eventual & 2,5 & 14,2 & \\
\hline Diferença de idade entre parceiros & $n=668$ & $\mathrm{n}=371$ & \\
\hline Parceiro/a mais novo/a & 0,6 & 27,5 & 0,0000 \\
\hline Mesma idade ( \pm 1 ano) & 19,7 & 48,1 & \\
\hline Parceiro/a mais velho/a (2-4 anos) & 37,6 & 14,6 & \\
\hline Parceiro/a muito mais velho/a ( 5 anos e + ) & 42,2 & 9,8 & \\
\hline Com quem morava & $n=683$ & $\mathrm{n}=377$ & \\
\hline Família de origem & 74,2 & 86,6 & 0,0145 \\
\hline Parceiro/a & 15,8 & 6,4 & \\
\hline Parceiro/a e familiares & 6,1 & 1,4 & \\
\hline Outros & 3,9 & 5,7 & \\
\hline Intenção de engravidar & $n=678$ & $\mathrm{n}=377$ & \\
\hline Estava tentando engravidar & 15,2 & 5,7 & 0,0004 \\
\hline Q ueria engravidar mais tarde & 14,4 & 8,7 & \\
\hline $\mathrm{N}$ ão queria engravidar & 37,7 & 42,1 & \\
\hline Não pensava no assunto & 32,6 & 43,5 & \\
\hline Uso de contracepção & $\mathrm{n}=682$ & $\mathrm{n}=376$ & \\
\hline $\operatorname{sim}$ & 31,4 & 36,3 & 0,3018 \\
\hline Não & 68,6 & 63,7 & \\
\hline Resultado da gravidez & $n=683$ & $\mathrm{n}=375$ & \\
\hline Filho & 72,2 & 34,5 & 0,0000 \\
\hline Aborto espontâneo & 9,6 & 18,4 & \\
\hline Aborto provocado & 15,3 & 41,3 & \\
\hline Está grávida & 2,9 & 5,7 & \\
\hline
\end{tabular}

* Relacionamento com 3 meses e mais.

Determinantes sociais da gravidez na adolescência

A ocorrência de uma gravidez antes dos vinte anos variou inversamente com a renda e a escolaridade (Tabela 3). Entre as mulheres, os contrastes são mais expressivos e a prevalência entre as que tinham até primeiro grau incompleto $(59,6 \%)$ corresponde a 13 vezes o valor observado entre aquelas com nível superior de instrução (4,6\%). As diferenças entre grupos raciais e étnicos foram menos marcantes, embora se mantendo estatisticamente significantes. Constatou-se uma maior prevalência de gravidez na adolescência entre homens e mulheres 
Prevalência de gravidez antes dos 20 anos entre homens e mulheres (inclui virgens), segundo determinantes sociais selecionados. Porto Alegre, Rio de J aneiro e Salvador, Brasil, 2000.

\begin{tabular}{|c|c|c|c|c|c|c|}
\hline \multirow[t]{2}{*}{ Características sociodemográficas } & \multicolumn{3}{|c|}{ Mulheres } & \multicolumn{3}{|c|}{ Homens } \\
\hline & $\mathrm{n}$ & $\%$ & $\mathrm{p}$-valor & $\mathrm{n}$ & $\%$ & $p$-valor \\
\hline \multicolumn{7}{|l|}{ Renda familiar per capita* } \\
\hline Muito baixa (até $\mathrm{R} \$ 90,00$ ) & 381 & 50,4 & 0,0000 & 267 & 33,9 & 0,0000 \\
\hline Baixa (de $\mathrm{R} \$ 91,00$ a $\mathrm{R} \$ 180,00$ ) & 487 & 36,8 & & 396 & 27,5 & \\
\hline Intermediária (de $\mathrm{R} \$ 181,00$ a $\mathrm{R} \$ 540,00$ ) & 833 & 24,4 & & 800 & 16,8 & \\
\hline Alta (Acima de $R \$ 540,00$ ) & 734 & 7,8 & & 712 & 11,2 & \\
\hline \multicolumn{7}{|l|}{ Escolaridade } \\
\hline 1o Grau incompleto & 437 & 59,6 & 0,0000 & 496 & 34,4 & 0,0000 \\
\hline 1o Grau completo & 575 & 36,8 & & 565 & 32,2 & \\
\hline 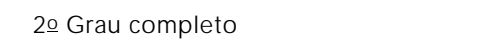 & 796 & 17,0 & & 635 & 13,1 & \\
\hline Superior incompleto/completo & 597 & 4,6 & & 447 & 6,5 & \\
\hline \multicolumn{7}{|l|}{ Raça/Cor } \\
\hline Branca & 1.165 & 20,5 & 0,0000 & 1.099 & 14,6 & 0,0000 \\
\hline Parda & 528 & 31,1 & & 442 & 17,3 & \\
\hline Preta & 550 & 37,4 & & 462 & 31,1 & \\
\hline Indígena & 133 & 30,8 & & 146 & 24,4 & \\
\hline \multicolumn{7}{|l|}{ Religião atual } \\
\hline Católica & 839 & 21,5 & 0,0030 & 589 & 20,3 & 0,2997 \\
\hline Pentecostal & 246 & 30,0 & & 178 & 22,4 & \\
\hline O utra & 451 & 28,0 & & 259 & 13,1 & \\
\hline Nenhuma & 882 & 33,6 & & 1.145 & 20,5 & \\
\hline \multicolumn{7}{|l|}{ Trabalho } \\
\hline Sim & 1.083 & 24,0 & 0,0066 & 1.317 & 21,6 & 0,2211 \\
\hline N ão & 1.352 & 30,6 & & 857 & 17,1 & \\
\hline
\end{tabular}

* Categorias definidas com base no salário mínimo nacional à época ( $R \$ 180,00)$.

negros, mas também entre pardos e indígenas, quando comparados àqueles que se declararam brancos (Tabela 3 ).

O efeito do pertencimento religioso só foi percebido entre as mulheres, sendo as católicas as que marcadamente declararam menor experiência de gravidez antes dos vinte anos (Tabela 3). Apenas entre as mulheres, observou-se uma associação inversa da ocorrência de gravidez na adolescência com a inserção no mercado de trabalho, sendo o evento mais freqüente entre as que não estavam trabalhando por ocasião da entrevista (Tabela 2).

Socialização para a sexualidade e a reprodução: as reiteradas diferenças de gênero

A entrada das mulheres na adolescência é demarcada pela menarca, evento biológico rein- terpretado pelos indivíduos, sendo a ele atribuídos significados culturais. A comunicação verbal entre mãe e filha em torno da menarca é uma oportunidade de transmissão de normas, valores e conhecimentos sobre comportamentos preventivos. As mulheres cujas mães conversaram sobre menstruação com elas antes da menarca - que corresponderam a $54,2 \%$ do total - engravidaram menos na adolescência (Tabela 4). Isso foi igualmente constatado quando a mãe e o pai foram incluídos entre as fontes das primeiras informações sobre gravidez e meios de evitar filhos, efeito não verificado entre os homens.

A maneira como os jovens recebem suas primeiras informações sobre reprodução e contracepção permite situá-los em diferentes perfis de socialização para a sexualidade, expressando, além disso, o peso das diversas instituições nesse processo. Além da família, a escola 
Prevalência de gravidez antes dos 20 anos de homens e mulheres (inclui virgens), segundo fatores de socialização para sexualidade e reprodução. Porto Alegre, Rio de J aneiro e Salvador, Brasil, 2000.

\begin{tabular}{|c|c|c|c|c|c|c|}
\hline \multirow[t]{2}{*}{ Indicadores de socialização } & \multicolumn{3}{|c|}{ Mulheres } & \multicolumn{3}{|c|}{ Homens } \\
\hline & $\mathrm{n}$ & $\%$ & $\mathrm{p}$-valor & $\mathrm{n}$ & $\%$ & $\mathrm{p}$-valor \\
\hline \multicolumn{7}{|c|}{$\begin{array}{l}\text { Participação nas tarefas domésticas } \\
\text { (entre } 15 \text { e } 18 \text { anos) }\end{array}$} \\
\hline Principal responsável & 361 & 54,4 & 0,0000 & 89 & 30,5 & 0,2832 \\
\hline Dividia igualmente com outros & 541 & 28,2 & & 350 & 20,7 & \\
\hline Ajudava & 1.119 & 22,5 & & 1047 & 20,1 & \\
\hline Não tinha nenhuma obrigação & 405 & 16,3 & & 684 & 17,1 & \\
\hline \multicolumn{7}{|c|}{ Conversa com a mãe sobre menstruação } \\
\hline Sim, antes da menarca & 1.289 & 21,4 & 0,0012 & NA & NA & \\
\hline Sim, após a menarca & 528 & 33,5 & & NA & NA & \\
\hline Nunca conversou & 560 & 35,1 & & NA & NA & \\
\hline \multicolumn{7}{|c|}{$\begin{array}{l}\text { Primeiras informações sobre gravidez } \\
\text { e contracepção }\end{array}$} \\
\hline \multicolumn{7}{|c|}{ Através da mãe } \\
\hline Sim & 1.125 & 23,6 & 0,0029 & 868 & 22,4 & 0,2545 \\
\hline Não & 1.308 & 30,6 & & 1.294 & 18,4 & \\
\hline \multicolumn{7}{|l|}{ Através do pai } \\
\hline Sim & 203 & 12,4 & 0,0001 & 607 & 18,3 & 0,4505 \\
\hline Não & 2.230 & 28,7 & & 1.555 & 20,3 & \\
\hline \multicolumn{7}{|l|}{ Através da escola } \\
\hline Sim & 874 & 19,4 & 0,0000 & 748 & 9,7 & 0,0012 \\
\hline Não & 1.559 & 31,7 & & 1.414 & 24,5 & \\
\hline \multicolumn{7}{|c|}{$\begin{array}{l}\text { Pais tentavam impedir a convivência com } \\
\text { certos amigos ou namorado/a (aos } 15 \text { anos) }\end{array}$} \\
\hline Sim & 998 & 35,3 & 0,0000 & 766 & 27,5 & 0,0000 \\
\hline Não & 1.419 & 21,5 & & 1.404 & 15,5 & \\
\hline
\end{tabular}

NA = Não se aplica.

cumpre um papel crucial na transmissão de conhecimentos, mas também de valores, em que pese as desigualdades de acesso ao sistema escolar no Brasil. Embora a maioria dos jovens de ambos os sexos não tenha mencionado a escola como fonte das primeiras informações sobre o tema, a prevalência de gravidez na adolescência foi significativamente mais baixa entre aqueles que a mencionaram (Tabela 4).

Outro indicador significativo do processo de socialização para a sexualidade é a interferência familiar no sentido de tentar impedir a convivência dos jovens com amigos e namorados/as aos quinze anos, o que reflete a existência de conflitos nessa área. No presente estudo, essa interferência mostrou-se associada à experiência mais freqüente de gravidez na adolescência, tanto para os homens quanto para as mulheres (Tabela 4). Também o acentuado encargo com tarefas domésticas por parte das meninas $-14,9 \%$ delas eram as principais responsáveis pelo desenvolvimento das mesmas assinala num claro gradiente a associação com a gravidez na adolescência (Tabela 4). Já os homens referiram menor envolvimento com as tarefas domésticas - 31,5\% não tinham qualquer obrigação sobre elas - não tendo sido confirmada uma associação desta variável com a experiência de engravidar uma parceira antes dos vinte anos.

Entrada na sexualidade e experiência reprodutiva

A entrada na sexualidade é um processo de experimentação física e relacional que se inicia antes e se estende até depois da primeira relação sexual. Nesse processo, são conformadas 
regras de comportamento e atribuídos status diferenciados aos parceiros, segundo os diversos tipos de relacionamento. Entre esses se incluem o namoro - mais ou menos tradicional e o "ficar" - modalidade recente e complementar, onde se pressupõe menor compromisso entre os parceiros. A maioria dos jovens experimentou ambas as modalidades $(89,7 \%$ dos homens e $72,5 \%$ das mulheres). No entanto, a gravidez na adolescência não foi constatada entre as mulheres que só "ficaram" com alguém, sendo, além disso, mais freqüente entre aquelas que declararam ter apenas namorado (Tabela 5). Entre os rapazes, a gravidez de uma parceira foi mais relatada pelos que experimentaram ambas as modalidades de relacionamento.

A ocorrência de gravidez na adolescência mostrou-se associada inversamente com a idade do primeiro namoro. Foi mais prevalente entre aqueles que tiveram iniciação sexual antes dos 15 anos, especialmente as mulheres. Entretanto, outros fatores podem explicar as diferenças entre os estratos, entre os quais se inclui o uso ou não de contracepção. O tipo de negociação entre parceiros na primeira relação pode ser expresso pelo relato sobre o modo como o uso de método contraceptivo foi ou não precedido de conversa. Assim sendo, a gravidez na adolescência foi expressivamente mais freqüente $(63,9 \%)$ entre mulheres que disseram não ter usado contracepção na iniciação sexual, nem conversado sobre o assunto com o parceiro, o que contrasta especialmente com o percentual observado entre as que conversaram e usaram contracepção (23,5\%) (Tabela 5). Quanto aos homens, a prevalência mais alta foi registrada entre os que conversaram sobre o assunto com a parceira, mas não usaram proteção; percentual equivalente foi observado quando os rapazes nem conversaram, nem usaram contracepção.

Chama a atenção entre as mulheres, a mais alta ocorrência de gravidez antes dos vinte anos quanto mais velho o parceiro da iniciação sexual, associação que não foi confirmada entre os homens (Tabela 5).

\section{Repercussões sociais da paternidade/ maternidade antes dos vinte anos}

O nascimento de um filho antes dos vinte anos motivou a constituição de uma união conjugal de $47,8 \%$ dos homens e $39,3 \%$ das mulheres, mas entre os primeiros, o percentual de união prévia $(9,3 \%)$ era inferior ao das últimas $(18,9 \%)$ (Tabela 6). De qualquer modo, é significativo que para $36 \%$ dos jovens de ambos os sexos o nascimento de um filho não tenha chegado a ocasionar uma saída da residência dos pais para constituição de união.

Durante o primeiro ano após o nascimento de um filho, $72,4 \%$ das mulheres, em contraste com $47,5 \%$ dos homens, declararam ter diminuído o convívio com o grupo de amigos (Tabela 6$)$.

Um aspecto interessante a ser contemplado diz respeito às mudanças e permanências na utilização de contracepção após o nascimento do primeiro filho na adolescência: $37,6 \%$ dos homens e $45,6 \%$ das mulheres passaram a usar algum método (Tabela 6). Todavia, respectivamente, $19,7 \%$ e $21,5 \%$ destes mantiveram-se em relações sexuais desprotegidas, sendo ainda mais expressivos os percentuais daqueles que engravidaram novamente, que corresponderam a uma razão de um em cada quatro jovens pais e mães adolescentes.

O nascimento de um filho antes dos vinte anos não pareceu afetar a situação escolar e de trabalho dos rapazes, exceto para $24,6 \%$ dos jovens pais que, no primeiro ano após o evento, pararam temporária ou completamente de estudar (Tabela 6). Entre as mães adolescentes, o impacto sobre o percurso escolar foi maior: $25,0 \%$ interromperam temporariamente e $17,3 \%$ definitivamente seus estudos no primeiro ano após o nascimento do filho. Contudo, $42,1 \%$ delas já se encontravam fora da escola por outros motivos, o que também ocorria em proporção ligeiramente superior $(48,7 \%)$ para os rapazes que tiveram o primeiro filho antes dos vinte anos.

\section{Discussão}

As taxas de resposta alcançadas nos três centros foram superiores àquelas alcançadas em outros inquéritos domiciliares sobre reprodução e sexualidade, realizados nas duas últimas décadas (BEMFAM/DHS, 1997; Laumann et al., 1994; Ministerio de la Salud, 2000), estando próximas àquelas obtidas em estudo nacional com população de faixa etária semelhante (BEMFAM, 1992). As perdas foram decorrentes, em geral, de dificuldades de acesso aos domicílios, principalmente em áreas reconhecidas como inseguras, onde não foi possível o retorno dos entrevistadores em horários noturnos, ou naquelas onde medidas de segurança adotadas pelos moradores impediram a entrada. Acredita-se que as dificuldades de acesso aos jovens, de todo não muito expressivas, deveram-se ao fato de que na faixa etária de interesse os sujeitos encontram-se com freqüência fora do domicílio, envolvidos em múltiplas atividades de es- 
Prevalência de gravidez antes dos 20 anos de homens e mulheres, segundo indicadores selecionados do modo de entrada na sexualidade. Porto Alegre, Rio de J aneiro e Salvador, Brasil, 2000.

\begin{tabular}{|c|c|c|c|c|c|c|}
\hline \multirow[t]{2}{*}{ Entrada na sexualidade } & \multicolumn{3}{|c|}{ Mulheres } & \multicolumn{3}{|c|}{ Homens } \\
\hline & $\mathrm{n}$ & $\%$ & $\mathrm{p}$-valor & $\mathrm{n}$ & $\%$ & $\mathrm{p}$-valor \\
\hline \multicolumn{7}{|l|}{ Experiência de ficar/namorar* } \\
\hline Ficou/não namorou & 48 & - & 0,0079 & 75 & 5,2 & 0,0015 \\
\hline Namorou/não ficou & 549 & 31,7 & & 147 & 9,6 & \\
\hline Namorou e ficou & 1.814 & 27,2 & & 1.930 & 21,5 & \\
\hline \multicolumn{7}{|l|}{ Idade ao primeiro namoro** } \\
\hline Até 13 anos & 726 & 40,7 & 0,0000 & 745 & 28,3 & 0,0000 \\
\hline $14-15$ anos & 1.000 & 28,7 & & 734 & 17,4 & \\
\hline 16 anos e mais & 634 & 10,4 & & 586 & 12,6 & \\
\hline \multicolumn{7}{|l|}{ Idade na iniciação sexual } \\
\hline Até 14 anos & 266 & 69,7 & 0,0000 & 583 & 33,5 & 0,0000 \\
\hline 15 anos e mais & 1.718 & 28,8 & & 1.442 & 16,4 & \\
\hline \multicolumn{7}{|l|}{$\begin{array}{l}\text { Conversa prévia e uso de contracepção } \\
\text { na iniciação sexual }\end{array}$} \\
\hline Conversaram e usaram & 1.027 & 23,5 & 0,0000 & 701 & 18,1 & 0,0018 \\
\hline Usaram sem conversar & 372 & 31,2 & & 722 & 16,3 & \\
\hline Conversaram e não usaram & 175 & 34,5 & & 107 & 34,8 & \\
\hline Nem conversaram, nem usaram & 384 & 63,9 & & 463 & 29,8 & \\
\hline \multicolumn{7}{|l|}{$\begin{array}{l}\text { Diferença de idade entre parceiros } \\
\text { da iniciação sexual }\end{array}$} \\
\hline Parceiro/a mais novo/a & 28 & 7,6 & 0,0008 & 186 & 15,5 & 0,4813 \\
\hline Mesma idade ( \pm 1 ano) & 461 & 25,5 & & 987 & 22,5 & \\
\hline Parceiro/a mais velho (2-4 anos) & 760 & 35,1 & & 432 & 24,2 & \\
\hline Parceiro/a muito mais velho ( 5 anos e +$)$ & 714 & 39,0 & & 281 & 21,7 & \\
\hline
\end{tabular}

* Excluído 1,0\% que nunca namorou, nem ficou.

** Excluídos 3,1\% que nunca namoraram.

tudo, trabalho e lazer. Todavia, em relação ao principal evento de interesse - a gravidez e a maternidade na adolescência - se houve algum viés de seleção, provavelmente isso ocorreu na direção da superestimação do fenômeno entre as mulheres, cuja condição implica a maior permanência em casa.

A experiência de gravidez na adolescência foi relatada de modo menos freqüente pelas jovens mulheres entrevistadas na Pesquisa Gravad do que o observado em estudos demográficos nacionais, realizados nas décadas de $80 \mathrm{e}$ 90 (BEMFAM, 1992, 1999; BENFAM/DHS, 1997). Isso ocorreu apesar de terem sido constatadas, nas três cidades, maiores proporções de moças com iniciação sexual do que o que se registrou em 1989/1990, no Rio de Janeiro, em Curitiba e em Recife na Pesquisa sobre Saúde Reprodutiva e Sexualidade do Jovem (BEMFAM, 1992), e para o conjunto do país em 1996 (BEMFAM, 1999).
As prevalências de gravidez na adolescência nas capitais brasileiras também foram menores do que as que podem ser inferidas em 43 países em desenvolvimento da África, Ásia, América Latina e Caribe, com base na comparação com proporções de mulheres de 20 a 24 anos que já tinham tido pelo menos um filho antes dos vinte e dos dezoito anos (Singh, 1998). Os valores estimados pela Pesquisa Gravad são próximos àqueles de países da América Latina, onde cerca de $1 / 3$ das mulheres já tinham pelo menos um filho antes dos 20 anos e 15,0 a 20,0\% delas o tiveram antes dos 18 anos. Porém, como as proporções apresentadas no estudo multipaíses se referem às mulheres com experiência de maternidade, não incluindo aquelas que só tiveram gestações terminadas em aborto, é permitido concluir que as diferenças em relação à ocorrência de gravidez na adolescência em Salvador, no Rio de Janeiro e em Porto Ale- 
Tabela 6

Distribuição de homens e mulheres que tiveram filho antes dos 20 anos segundo repercussões sociais no primeiro ano após o nascimento do primeiro filho. Porto Alegre, Rio de J aneiro e Salvador, Brasil, 2000.

\begin{tabular}{|c|c|c|c|}
\hline Repercussões & $\begin{array}{c}\text { Mulheres } \\
\%\end{array}$ & $\begin{array}{l}\text { Homens } \\
\%\end{array}$ & p-valor \\
\hline Mudança de residência & $n=512$ & $n=154$ & \\
\hline Permaneceu com a família & 36,4 & 36,1 & 0,2544 \\
\hline $\begin{array}{l}\text { Foi morar com a/o parceira/o } \\
\text { com/sem família }\end{array}$ & 39,3 & 47,8 & \\
\hline Já morava com a/o parceira/o & 18,9 & 9,3 & \\
\hline Outra & 5,4 & 6,9 & \\
\hline $\begin{array}{l}\text { Diminuição do convívio } \\
\text { com grupos de amigos }\end{array}$ & $n=508$ & $n=148$ & \\
\hline Sim & 72,4 & 47,5 & 0,0009 \\
\hline Não & 27,6 & 52,5 & \\
\hline $\begin{array}{l}\text { Em termos de uso de métodos } \\
\text { contraceptivos }\end{array}$ & $n=512$ & $\mathrm{n}=150$ & \\
\hline Passou a usar & 45,6 & 37,6 & 0,0439 \\
\hline Não usava e permaneceu assim & 16,8 & 16,5 & \\
\hline Usava e deixou de usar & 4,7 & 3,2 & \\
\hline Continuou usando o mesmo método & 6,9 & 3,5 & \\
\hline Engravidou novamente & 23,6 & 24,8 & \\
\hline O utro & 2,5 & 14,3 & \\
\hline Em termos de trabalho & $\mathrm{n}=513$ & $n=153$ & \\
\hline $\mathrm{N}$ ão estava trabalhando e manteve-se assim & 62,6 & 14,1 & 0,0000 \\
\hline Parou de trabalhar & 6,6 & 3,5 & \\
\hline Já trabalhava e permaneceu assim & 13,7 & 65,7 & \\
\hline Começou a trabalhar & 16,7 & 15,8 & \\
\hline Outra & 0,5 & 1,0 & \\
\hline Em termos de estudo & $n=513$ & $\mathrm{n}=153$ & \\
\hline Não estava estudando e manteve-se assim & 42,4 & 48,7 & 0,0054 \\
\hline Continuou estudando & 14,1 & 25,9 & \\
\hline Parou de estudar por um período & 25,0 & 8,8 & \\
\hline Parou completamente de estudar & 17,3 & 15,8 & \\
\hline O utro & 1,1 & 0,8 & \\
\hline
\end{tabular}

gre sejam ainda maiores. Todavia, estes valores são bem superiores àqueles oriundos de um estudo que compara a ocorrência do fenômeno em cinco países desenvolvidos, o qual registra proporções de mulheres entre 20 e 24 anos que já tinham tido filhos antes dos vinte anos, variando de 4,0\% na Suécia a 22,0\% nos Estados Unidos (Darroch et al., 2001). Mesmo considerando que as proporções apresentadas no último estudo não incluem aquelas que só tiveram gestações terminadas em aborto, ainda assim a experiência de gravidez na adolescência entre jovens brasileiras é bem mais freqüente.
No presente estudo, foi muito pequena a proporção de mulheres que tiveram uma gravidez antes dos 15 anos, o que é consistente com o observado em países desenvolvidos (Darroch et al., 2001), e mesmo naqueles em desenvolvimento, exceto na África Subsaariana, onde os percentuais situaram-se de 8,0 a $15,0 \%$ (Singh, 1998). Em que pese a pequena magnitude, a ocorrência de uma gravidez nessa faixa etária não deixa de ser importante pelo maior risco de complicações obstétricas e pela possibilidade de estar associada à violência sexual (Stern \& Garcia, 1999), o que poderá ser mais bem investigado à medida que a análise de dados seja aprofundada. Entretanto, não é possível deixar de enfatizar que, nas capitais brasileiras, a grande maioria das gestações em sua primeira vez ocorreu aos 18 e 19 anos, quando as jovens já tinham atingido o marco da maioridade civil, ainda que mantendo relações de dependência econômica e domiciliar com suas famílias de origem. De fato, a maioria das gestações ocorreu fora de uniões conjugais, como já havia sido identificado anteriormente (Longo \& Rios Neto, 1998), em parte motivadas pela perspectiva do nascimento de um filho; mas sua quase totalidade se deu no contexto de relacionamentos estáveis, onde a gravidez era de certa forma um risco assumido, o que se reflete na baixa utilização de contraceptivos após a iniciação sexual. Isso tem sido identificado em outros estudos (Almeida et al., 2003; BEMFAM/ DHS, 1997; Brandão et al., 2001), e merecerá um exame mais criterioso da questão.

A análise comparativa dos relatos dos homens é ainda mais difícil, em decorrência da escassez absoluta de estudos que enfoquem a experiência masculina de ter engravidado uma parceira nessa etapa (Lyra, 1998). Os dados provenientes de pesquisas demográficas nacionais permitem constatar que a ocorrência do fenômeno na Pesquisa Gravad foi menos freqüente do que a média nacional de $30,5 \%$, constatada em 1996, entre jovens de 20 a 24 anos (BEMFAM, 1999), mas não é possível destacar deste percentual aqueles onde as gestações ocorreram antes dos vinte anos.

A incorporação da perspectiva dos parceiros masculinos evidencia ser incompleta a compreensão da ocorrência da gravidez na adolescência sem uma abordagem sistemática do cenário das relações de gênero. Essas integram não somente o modo de socialização familiar por exemplo, na conversa entre mãe e filha sobre menarca e na realização de tarefas domésticas -, o controle parental acentuado sobre o namoro e a diminuição do convívio social com o nascimento de um filho, bem como a diferen- 
ça de idades entre parceiros, que exprime uma assimetria de posições entre os gêneros.

O uso de contracepção é um aspecto crucial na análise do objeto em foco, e os resultados apresentados já permitem confirmar uma tendência ao relaxamento nas práticas de proteção para a gravidez na medida em que se consolidam relacionamentos mais estáveis, o que tem sido apontado em outros estudos (Almeida et al., 2003; BEMFAM/DHS, 1997), e também foi constatado na etapa qualitativa da Pesquisa Gravad (Brandão et al., 2001). As relações de gênero enquadram o exercício da sexualidade e o possível controle da fecundidade. Embora a contracepção persista sendo considerada um assunto feminino, a conversa sobre o tema entre parceiros revela a importância que a proteção adquire no desenrolar da experimentação sexual a dois, indicando o interesse sobre as conseqüências do ato sexual. Entretanto, seu uso efetivo dependerá de múltiplos fatores (Almeida et al., 2003) e, certamente, a assimetria de gênero, redobrada pela diferença de idade entre parceiros, se reflete na capacidade de negociação acerca da contracepção, especialmente no momento da iniciação sexual, o que deverá ser mais bem explorado em outras publicações.

A assimetria de gênero vai se expressar ainda de modo mais contundente quando se enfocam as repercussões sociais de uma gravidez na adolescência, sendo seu impacto mais evidente nas carreiras escolares das moças, o que também deverá ser mais bem investigado com o aprofundamento da análise. Diversos autores têm ressaltado as dificuldades envolvidas na análise da relação entre precocidade do início da vida reprodutiva e desempenho escolar (Brandão, 2003; Hoffman, 1998; Stern \& Garcia, 1999). Assim como constatado no presente estudo, inúmeras pesquisas têm evidenciado a existência de uma relação inversa entre escolaridade e reprodução na adolescência, sendo, entretanto, difícil estabelecer a existência de uma relação de causalidade em qualquer direção (Gupta \& Leite, 1999; Hoffmann, 1998; Stern \& Garcia, 1999).

No Brasil, existe uma grande heterogeneidade das trajetórias juvenis, particularmente quanto ao processo de escolarização e à inserção no mercado de trabalho (Sabóia, 1998). Entre os grupos sociais mais favorecidos, observa-se um prolongamento da juventude com o aumento do tempo de estudo, a manutenção da coabitação com os pais e a aquisição tardia de autonomia material (Brandão et al., 2001). Nas classes populares, a juventude tende a ser mais breve com a interrupção precoce dos estudos e a inserção (ainda que precária) no mercado de trabalho, sem que seja atingida uma autonomia plena, em decorrência da precariedade das condições de vida (Heilborn et al., 2002). Entretanto, as desigualdades de classe social articulam-se às de gênero, reservando distintos papéis sociais aos jovens homens e mulheres, como foi possível constatar no presente estudo. A realização de tarefas domésticas pelas mulheres inicia-se muito cedo, sendo parte do processo de socialização para a maternidade, especialmente entre as classes populares (Brandão, 2003). As trajetórias escolares femininas, ainda que mais longas do que as masculinas, são igualmente descontínuas, com grande defasagem idade/série e interrupções que antecedem a ocorrência de gravidez ou nascimento de um filho. Em contextos fortemente marcados por desigualdades de gênero e classe social, a maternidade se apresenta não apenas como "destino", mas como fonte de reconhecimento social (Le Van, 1998), para as jovens mulheres, desprovidas de projetos educacionais e profissionais.

\section{Agradecimentos}

O projeto Gravidez na Adolescência: Estudo Multicêntrico sobre Jovens, Sexualidade e Reprodução no Brasil foi originalmente elaborado por Maria Luiza Heilborn (Programa em Gênero, Sexualidade e Saúde, Instituto de Medicina Social, Universidade do Estado do Rio de Janeiro), Michel Bozon (Institut National d'Études Démographiques), Estela M. L. Aquino (Programa de Estudos em Gênero e Saúde, Instituto de Saúde Coletiva, Universidade Federal da Bahia), Daniela Knauth e Ondina Fachel Leal (Núcleo de Pes- quisa em Antropologia do Corpo e da Saúde, Universidade Federal do Rio Grande do Sul). Agradecemos a Ceres G. Victora, Fabíola Rohden, Cecilia McCallum, Tania Salem, Elaine Brandão e aos demais membros da equipe Gravad, bem como ao consultor estatístico do estudo, Antônio José Ribeiro Dias (Fundação Instituto Brasileiro de Geografia e Estatística). Agradecemos, ainda, o apoio financeiro da Fundação Ford e ao Programa de Bolsas do Conselho Nacional de Desenvolvimento Científico e Tecnológico. 


\section{Referências}

ALMEIDA, M. C. C.; AQUINO, E. M. L.; GAFFKIN, L. \& MAGNANI, R. J., 2003. Uso de contracepção por adolescentes de escolas públicas na Bahia. Revista de Saúde Pública, 37:566-575.

BEMFAM (Sociedade Civil Bem-estar Familiar no Brasil), 1992. Pesquisa sobre Saúde Reprodutiva e Sexualidade do Jovem. Rio de Janeiro, Curitiba e Recife. 1989/90. Rio de Janeiro: BEMFAM/Centers for Disease Control and Prevention.

BEMFAM (Sociedade Civil Bem-estar Familiar no Brasil), 1999. Adolescentes, Jovens e a Pesquisa Nacional sobre Demografia e Saúde. Rio de Janeiro: BEMFAM.

BEMFAM (Sociedade Civil Bem-estar Familiar no Brasil)/DHS (Demography and Health Survey), 1997. Brasil. Pesquisa Nacional sobre Demografia e Saúde. 1996. Rio de Janeiro: BEMFAM/DHS.

BRANDÃO, E. R., 2003. Individualização e Vínculo Familiar em Camadas Médias: Um Olhar Através da Gravidez na Adolescência. Tese de Doutorado, Rio de Janeiro: Instituto de Medicina Social, Universidade do Estado do Rio de Janeiro.

BRANDÃO, E. R.; HEILBORN, M. L.; AQUINO, E. M. L.; KNAUTH, D. R. \& BOZON, M., 2001. Juventude e família: Reflexões preliminares sobre a gravidez na adolescência em camadas médias urbanas. Intersecções: Revista de Estudos Interdisciplinares, 3:159-180.

CAMARANO, A. A., 1998. Fecundidade e anticoncepção da população de 15-19 anos. In: Seminário Gravidez na Adolescência, Anais, pp. 35-46, Rio de Janeiro: Ministério da Saúde/Family Health International/Associação Saúde.

DARROCH, J. E.; SINGH, S. \& FROST, J. J., 2001. Differences in teenage pregnancy rates among five developed countries: The roles of sexual activity and contraceptive use. Family Planning Perspectives, 33:244-250.

GALLAND, O., 1995. Sociologie de la Jeunesse. Paris: Armand Collin.

GUPTA, N. \& LEITE, I. C., 1999. Adolescent fertility behaviour: Trends and determinants in Northeastern Brazil. International Family Planning Perspectives, 25:125-130.

HEILBORN, M. L.; SALEM, T.; KNAUTH, D. R.; AQUINO, E. M. L.; BOZON, M.; ROHDEN, F.; VICTORA, C.; McCALLUM, C. \& BRANDÃO, E. R., 2002. Aproximações socioantropológicas sobre a gravidez na adolescência. Horizontes Antropológicos, 8:13-45.
HOFFMANN, S. D., 1998. Teenage childbearing is not so bad after all...or is it? A review of the new literature. Family Planning Perspectives, 30:236-243.

LAUMANN, E. O.; GAGNON, J. H.; MICHAEL, R. T. \& MICHAELS, S., 1994. The Social Organization of Sexuality: Sexual Practices in the United States. Chicago: University of Chicago Press.

LE VAN, C., 1998. Les Grossesses à l'Adolescence. Normes Sociales, Realités Vecues. Paris: L'Harmattan.

LONGO, L. F. B. \& RIOS NETO, E., 1998. Virgindade Matrimonial e Iniciação Sexual: Uma Análise Temporal. XI Encontro Nacional de Estudos Populacionais. 31 Outubro 2003 <http://www.abep.nepo. unicamp.br/docs/anais/PDF/1998/a131.pdf>.

LYRA, J., 1998. A Construção Social da Invisibilidade da Paternidade Adolescente nos Dados Demográficos. XII Encontro Nacional de Estudos Populacionais. <http://www.abep.org.br>.

MINISTERIO DE LA SALUD, 2000. Estudio Nacional de Comportamiento Sexual: Primeros Análisis, Chile, 2000. Santiago de Chile: Ministerio de la Salud.

MS (Ministério da Saúde), 1999. Saúde e Desenvolvimento da Juventude Brasileira: Construindo uma Agenda Nacional. Brasília: MS.

SABÓIA, A. L., 1998. Situação educacional dos jovens. In: Jovens Acontecendo na Trilha das Políticas Públicas (Comissão Nacional de População e Desenvolvimento - CNPD, org.), pp. 499-518, Brasília: CNPD.

SINGH, S., 1998. Adolescent childbearing in developing countries: A global review. Studies in Family Planning, 29:117-136.

STERN, C. \& GARCIA, E., 1999. Hacia un Nuevo Enfoque en el Campo del Embarazo Adolescente. Reflexiones. Sexualidad, salud y reproducción 13. México, DF: Programa Salud Reproductiva y Sociedad/El Colegio de México.

STERN, C. \& MEDINA, G., 2000. Adolescencia y salud en México. In: Cultura, Adolescência e Saúde: Argentina, Brasil e México (M. C. Oliveira, org.) pp. 98-160, Campinas: Consórcio de Programas em Saúde Reprodutiva e Sexualidade na América Latina.

WHO (World Health Organization), 1986. Young People's Health - A Challenge for Society. World Health Organization Technical Report Series 731. Geneva: WHO.

Recebido em 31 de outubro de 2003

Aprovado em 11 de novembro de 2003 